\title{
Inflow Boundary Conditions in Quantum Transport Theory
}

\author{
HORST LANGE $^{\mathrm{a}}$, BRUCE TOOMIRE ${ }^{\mathrm{b}}$ and P. F. ZWEIFEL ${ }^{\mathrm{b}, *}$ \\ ${ }^{a}$ Fachbereich Mathematik, Universität Köln Weyertal 86-90 D-50931 Köln, Germany; ${ }^{\mathrm{b}}$ Physics Department, \\ Virginia Polytechnic Institute and State University, Blacksburg, VA 24061-0435, USA
}

(Received 13 August 1997; In final form 1 December 1998)

\begin{abstract}
A linear (given potential) steady-state Wigner equation is considered in conjunction with inflow boundary conditions and relaxation-time terms. A brief review of the use of inflow conditions in the classical case is also discussed. An analytic expansion of solutions is shown and a recursion relation derived for the given problem under certain regularity assumptions on the given inflow data. The uniqueness of the physical current of the solutions is shown and a brief discussion of the lack of charge conservation associated with the relaxation-time is given.
\end{abstract}

Keywords: Inflow, boundary, conditions, steady-state, Wigner

\section{INTRODUCTION}

In the development of various types of semiconductor devices, the crucial information needed by the design engineer is the $I-V$ curve, i.e., the current flowing through the device as a function of the applied voltage. Typically, such devices are nonohmic and can, in fact, exhibit negative differential resistance, i.e., as the voltage increases through a certain range, the current can decrease ( $c f$. Fig. 1, Ref. [1]).

During the past decade, interest in quantum semi-conductors has been particularly intense due to the development of devices which depend upon quantum phenomena for their operation (e.g., resonance-tunneling diodes) $[1,2]$ as well as to the emergence of microscopic devices (e.g., "quantum dots") [3] in which quantum mechanics enters due to the omnipresence of boundaries.

To deal with quantum semi-conductors a popular approach has been the Wigner equation $(W E)$ or, more generally, the Wigner-Poisson (WP) system of equations [4]. $W E$ refers to a linear system, i.e., with a given potential, while $W P$ involves a selfconsistent Coulomb force. These systems may be considered quantum versions of the classical Vlasov transport equation (or Vlasov-Poisson system in the nonlinear case).

Many studies of WP have been carried out for the whole space $[5,6]$. But for modeling semi-conductors, one must deal with finite geometry, and hence the problem of boundary conditions must be

\footnotetext{
*Corresponding author.
} 
faced. Periodic boundary conditions were considered in Ref. [7]. (See the bibliography there for further references).

Since the Wigner equation is supposed to be simply a reformulation of quantum mechanics as expressed by the Schrödinger equation [4], one would expect that the appropriate boundary conditions for the former should be those induced, through the definition of the Wigner function, by self-adjoint boundary conditions on the quantum hamiltonian. Examples of such boundary conditions are Floquet $\left(\psi(x+1)=\mathrm{e}^{i \alpha} \psi(x), \alpha \in \mathbb{R}\right)$; Neumann and Dirichlet. (Note $\alpha=0$ in the Floquet case corresponds to the periodic boundary conditions mentioned earlier).

None of these conditions, for one reason or another, can be lifted satisfactorily to the Wigner equation (see Ref. [8] for a complete discussion); thus something else must be sought, for example the inflow conditions used in Refs. [1] and [2]. A modified version of the inflow conditions, socalled absorbing boundary conditions $[9,10,11]$ are sometimes used to avoid specious reflections at boundaries, but such conditions will not be considered here. To try to understand the (nonquantum) origin of inflow conditions, recall [4] the formula connecting the Wigner function $w(x, v, t)$ with the Schrödinger wave-function $\psi$ (in the onedimensional case):

$$
\begin{array}{r}
w(x, v, t)=\frac{1}{2 \pi} \int_{\mathbb{R}} \mathrm{e}^{i \eta v} \bar{\psi}\left(x+\frac{\eta}{2}, t\right) \times \\
\times \psi\left(x-\frac{\eta}{2}, t\right) d \eta
\end{array}
$$

(See also [4] for the definition of the Wigner transform of an operator $A$ ). Inflow conditions at $x=0$ (specification of $w(0, v, t)$ for $v>0)$ arise from a pure incoming wave

$$
\int w_{+}(v) \mathrm{e}^{i v x} d v, \quad v>0
$$

for all $x$, not solely for $x<0$. So inflow conditions are definitely non-quantum.
Nonetheless we have decided to use the inflow conditions [9]. These, common in transport theory, specify the incoming distribution of particles at free surfaces. We take the point of view that the Wigner equation is an entity unto itself separately from its origin in the Schrödinger equation, and seek to determine whether or not it is well-posed with inflow conditions. In Ref. [12] this problem was treated for the time-dependent case, with homogeneous inflow conditions, while in Ref. [13] the existence of unique mild solutions for the time-dependent nonlinear equation with inhomogeneous inflow data was proved for a crystal lattice with the velocity restricted to the first Brillouin Zone. In Ref. [14], existence of solutions to the nonlinear Schrödinger equation is proved for scattering states. A "cutoff" Wigner function is then defined which, in the $\hbar \rightarrow 0$ limit, is shown to tend (in the sense of distributions) to the solution of the stationary Vlasov equation with inflow boundary conditions.

In this paper, we consider the linear timedependent and stationary equations (with emphasis on the latter) for both bounded and unbounded velocity domains.

In order to help clarify the situation for $W E$, we begin (in Section II) with a brief discussion of the classical linear Vlasov equation $(V E)$, and continue with the non-stationary $W E$ in Section III. Adopting a relaxation-time model [15] for the time-dependent equation, we are able to show exponential decay to a solution of the stationary equation. In Section III, we discuss solutions of the stationary $W E$; they are known to exist, and they are called BGK modes [16] in the case of $V E$, and QBGK modes for $W E$ [17]. They are not unique. In fact, the non-uniqueness corresponds to "trapped particles" in the classical case [18] and the analogue thereof in the quantum case (bound states). We have been able to prove the existence of a weak solution for the stationary equation with inflow boundary conditions, but the current may not be defined unless the solution possesses sufficient additional regularity in which case the current exists and is constant. If we restrict our 
attention to the first Brillouin Zone, as in Ref. [13], we obtain strong solutions and unique existence of the current. In the Appendix we discuss some deficiencies of the relaxation time model and discuss a better model. We hope eventually to detail results for $V P$ and $W P$ similar to those presented here. In fact, it is not even obvious that the linear Wigner equation has any advantage over the ordinary Schrödinger theory, so this paper might be considered a first step toward dealing with the (nonlinear) $W P$ system.

\section{REMARKS ON THE LINEAR VLASOV EQUATION}

Henceforth we restrict our attention to one-dimensional systems as representing reasonable models of most semiconductor devices. Our remarks on the $V E$ problem are motivated by the fact that we use some similar ideas for the Wigner equation. The relevant equation, with a relaxation-time scattering model, can be written (with the electron charge $=-1$ )

$$
\frac{\partial f}{\partial t}+v \frac{\partial f}{\partial v}+V^{\prime}(x) \frac{\partial f}{\partial v}+\frac{f}{\tau}=\frac{f_{0}}{\tau} .
$$

Here $f=f(x, v, t), x \in[0,1], v \in \mathbb{R}, t \in \mathbb{R}^{+} ; \tau$ is the relaxation time. The relaxation distribution, $f_{0}(x, v)$ is assumed to obey the corresponding stationary equation.

$$
v \frac{\partial f_{0}}{\partial x}(x, v)+V^{\prime}(x) \frac{\partial f_{0}}{\partial v}=0,
$$

with $f_{0} \in L^{2}\left([0,1] \times \mathbb{R}_{v}\right)$.

Arnold [15] has already noted that $f_{0}$ should be position-dependent. In the solid-state physics literature [19] (and in Ref. [9]) $f_{0}$ is taken constant in space. Equation (2.1) is to be solved subject to the conditions

$$
\begin{gathered}
f(x, v, 0)=f_{I}(x, v) \\
f(0, v, t)=f_{I}(0, v)=f_{+}(v) \quad v>0
\end{gathered}
$$

$$
f(1, v, t)=f_{I}(1, v)=f_{-}(v) \quad v<0
$$

with $f_{I} \in X, f_{+}, f_{-} \in L^{2}\left(\mathbb{R}_{v}\right)$ where solutions are sought in the space

$$
X=L^{2}\left([0,1] \times \mathbb{R}_{v}\right) .
$$

Assuming sufficient regularity, for instance strong solutions which lie in the domain of the linear operator

$$
A=v \frac{\partial}{\partial x}+V^{\prime}(x) \frac{\partial}{\partial v}
$$

one proves without too much difficulty

Proposition 2.1 There exists a unique strong solution of Eq. (2.1) subject to (2.3)-(2.5).

Proposition 2.2 There exist classical solutions $f_{0} \in D(A)$ to the stationary Eq. (2.2) subject to (2.4) and (2.5).

The proof of Proposition 2.2 involves the introduction of the BGK modes [16], i.e., arbitrary functions of the energy. (All solutions of (2.2) are BGK modes). The energy, in turn, generates a set of characteristics to (2.2) which determine the solution uniquely on the non-periodic orbits originating from the inflow data. On the periodic characteristics, any function of the energy with sufficient regularity and compact support is a solution. These periodic solutions represent "trapped particles" [18] and are independent of the inflow. The solution is unique modulo these trapped particle solutions.

Proposition 2.3 If $f, f_{I}, f_{0} \in D(A)$ then $f(\cdot, \cdot, t)$ $\rightarrow f_{0}$ in $X$ as $t \rightarrow \infty$ where $f$ is a strong solution to (2.1) and $f_{0}$ a classical solution to (2.2).

Proposition 2.4 Let $v f_{0} \in L^{1}\left(\mathbb{R}_{v}\right)$. Then the stationary current calculated from the $f_{0}$ of Proposition 2.2 is unique.

Proof We have already pointed out that the solution is unique modulo trapped particles which obviously cannot contribute to the current, so the result is intuitively correct. It can be proved by 
integrating Eq. (2.2) over $v$; then since the current is given by

$$
I(x)=-\int_{\mathbb{R}_{v}} v f_{0}(x, v) d v
$$

it follows that

$$
\frac{\partial I(x)}{\partial x}=0
$$

the current is constant. Now take two different solutions with the same inflow data, $f_{0}^{(1)}$ and $f_{0}^{(2)}$. Then

$$
f_{0}=f_{0}^{(1)}-f_{0}^{(2)}
$$

satisfies (2.2) with zero inflow. Multiply by $f_{0}$ and integrate to obtain

$$
\int_{\mathbb{R}_{v}} v\left[f_{0}^{2}(1, v)-f_{0}^{2}(0, v)\right] d v=0 .
$$

Since both terms are non-negative (by the zero inflow condition) both vanish.

Thus, for example,

$$
f_{0}^{(1)}(1, v)=f_{0}^{(2)}(1, v)
$$

or from $(2.8)$

$$
I^{(1)}(1)=I^{(2)}(1)
$$

and since the current is constant, the proposition follows.

The above proof makes it clear that the current can be calculated from the solution for $v>0$ ). (We obtain a similar result for the Wigner equation in Section IV). In fact, in the classical case, the inflow data alone determine the current:

$I=-\int_{\sqrt{2 V_{\max }}}^{\infty} v f_{+}(v) d v-\int_{-\sqrt{2 V_{\max }}}^{-\infty} v f_{-}(v) d v$

for $V_{\max }>0$, where $V_{\max }$ is the maximum of the potential on $[0,1]$. This equation can sometimes be used as a figure of merit for the degree of "quantumness" of a semiconductor system;
Eq. (2.10) does not hold for quantum systems due to tunneling and quantum reflection.

\section{THE WIGNER EQUATION}

The equation we deal with is $[4,5,6]$

$$
\begin{array}{r}
\frac{\partial f}{\partial t}(x, v, t)+v \frac{\partial f}{\partial x}-i \Theta(V) f(x, v, t) \\
+\frac{f}{\tau}=\frac{f_{0}}{\tau}, \quad x \in[0,1] .
\end{array}
$$

The velocity variable $v \in \mathbb{R}$ (Case 1 ) or $v \in B$, the first Brioullin zone (Case 2). As is pointed out in Ref. [13], defining the density (and, we should mention, the current) in Case 1 is "a major problem since $L^{1}$ estimate is usually not available." While this statement refers to the nonlinear problem, it appears to be equally valid for the linear case.

The definition of the pseudo-differential operator $\Theta(V)$ is, for Case 1 ,

$$
\begin{aligned}
(\Theta(V) f)(x, v, t)=\frac{1}{2 \pi} & \int_{\mathbb{R}_{v}^{\prime} \times \mathbb{R}_{k}} \mathrm{e}^{i k\left(v-v^{\prime}\right)} \times \\
& \times\left[V\left(x+\frac{k}{2}\right)\right. \\
& \left.-V\left(x-\frac{k}{2}\right)\right] f\left(x, v^{\prime}, t\right) d v^{\prime} d k
\end{aligned}
$$

while for Case 2 it is defined as in Eq. (1.10) of Ref. [13]. Note that the potential must be extended beyond the interval $[0,1]$ for $(3.2)$ to make sense.

Analogously to Section II, in (3.1) $f_{0}$ is taken to be a solution of the stationary Wigner equation

$$
v \frac{\partial f_{0}(x, v)}{\partial x}+i \Theta(V) f_{0}=0
$$

Equation (3.1) is subject to conditions (2.3) - (2.5) and Eq. (3.3) is subject to (2.4) and (2.5).

In Ref. [7], the momentum was quantized due to the periodic boundary conditions. In the present, inflow, case no quantization is indicated, again 
verifying that our model, which does not derive from self-adjoint boundary conditions on a quantum hamiltonian, is at best quasi-classical [8]. The following results are formulated for Case 1, but are equally valid for Case 2 , with $\mathbb{R}_{v}$ replaced by $B$.

LEMmA $3.1 \Theta(V)$ is a bounded skew-adjoint operator on $X$. If $\Theta f(x, \cdot, t) \in L^{1}\left(\mathbb{R}_{v}\right)$, then

$$
\int(\Theta f)(x, v, t) d v=0
$$

Proof See Lemmata 1 and 2 of Ref. [12] for the first statement. The second follows directly from integration of (3.3) after an obvious variable transformation.

Remark The skew-adjoint property of $\Theta$ implies

$$
\int f(x, v, t)(\Theta f)(x, v, t) d v=0 .
$$

Introduce the operator $\Omega$ defined by

$$
\Omega f=v \frac{\partial f}{\partial x}+i \Theta(V) f
$$

defined on

$$
\begin{aligned}
D(\Omega)= & \left\{f \in X \mid v \frac{\partial f}{\partial x} \in X, f\right. \text { satisfies } \\
& \text { homogeneous inflow conditions }\}
\end{aligned}
$$

Then

Proposition $3.2 \Omega$ generates a continuous semigroup of contractions $T(t)$ on $X$.

Proof It is proved in Lemma 6 of Ref. [12] that $-v(\partial / \partial x)$ generates a contraction semigroup on $X$. Since (Lemma 3.1) $i \Theta(V)$ is a bounded perturbation to $-v(\partial / \partial x)$, the result follows (see Ref. [20]).

Introduce the operator

$$
C f=v \frac{\partial f}{\partial x}, \quad D(C)=\{f \in X \mid C f \in X\}
$$

We define a strong solution of (3.1) to be a function $\left.f \in C([0, T] ; D(C)) \cap C^{1}([0, T] ; X)\right]$, where $D(C)$ is equipped with the graph norm, such that $f$ obeys Eqs. (2.3) - (2.5). Then

Proposition 3.3 A strong solution of (3.1) is unique.

Proof Assume, by way of contradiction, that there are two solutions $f_{1}$ and $f_{2}$ with the same data, and set $f=f_{1}-f_{2}$. Then $f$ obeys

$$
\frac{\partial f}{\partial t}+v \frac{\partial f}{\partial t}+i \Theta(V) f+\frac{f}{\tau}=0
$$

subject to zero initial and homogeneous inflow data. Multiply (3.9) by $f$ and integrate over $x, v, t$ to obtain

$$
\begin{aligned}
\frac{1}{2}\|f(\cdot, \cdot, T)\|_{X}^{2} & +\int_{0}^{T} \int_{\mathbb{R}_{v}} v\left[f^{2}(1, v, t)\right. \\
& \left.-f^{2}(0, v, t)\right] d v d t \\
& +\frac{1}{\tau} \int_{0}^{T}\left\|f^{2}(\cdot, \cdot, t)\right\|_{X} d t=0 .
\end{aligned}
$$

(All operations used in obtaining (3.10) are allowed by the definition of strong solutions and by the fact that $v f(x, v, t)$ is a continuous function in $X$ as is implied by Sobolev imbedding of $v f$ ).

Since in (3.10) all terms are non-negative, it follows that $f=0$, proving the result.

We seek solutions of (3.1) in the form

$$
f(x, v, t)=f_{h}(x, v, t) \mathrm{e}^{-t / \tau}+f_{p}(x, v)
$$

where $f_{h}$ is a strong solution of the homogeneous equation

$$
\frac{\partial f_{h}}{\partial t}(x, v, t)+v \frac{\partial f_{h}}{\partial x}+i \Theta(V) f_{h}=0
$$

with

$$
f_{h}(x, v, 0)=f_{I}(x, v)-f_{p}(x, v)
$$


and homogeneous inflow data

$$
\begin{aligned}
& f_{h}(0, v, t)=0, \quad v>0 \\
& f_{h}(1, v, t)=0, \quad v<0
\end{aligned}
$$

Substituting into (3.1) gives the following equation for $f_{p}$ :

$v \frac{\partial f_{p}}{\partial x}(x, v)-i \Theta(V) f_{p}=\frac{f_{0}(x, v)-f_{p}(x, v)}{\tau}$,

with boundary conditions

$$
\begin{aligned}
& f_{p}(0, v)=f_{+}(v), \quad v>0 \\
& f_{p}(1, v)=f_{-}(v), \quad v<0
\end{aligned}
$$

with $v\left(\partial f_{p} / \partial x\right) \in X$. We note that $f_{p}=f_{0}$ solves (3.16)-(3.18).

Proposition 3.4 Let $f_{I}-f_{p} \in D(\Omega)$. Then there exists a unique global strong solution $f_{h}$ to the system (3.12)-(3.15) such that $e^{-t / \tau} f_{h}(\cdot, \cdot, t) \rightarrow 0$ in $X$ as $t \rightarrow \infty$.

Proof The global existence and uniqueness follow from Proposition 3.3; uniform boundedness of $f_{h}$ in $t$ on $X$ is implied by the following argument. Multiply Eq. (3.12) by $f_{h}$ and integrate over $x, v, t$ to obtain

$$
\begin{aligned}
\left\|f_{h}(\cdot, \cdot, t)\right\|_{X}^{2}+ & \int_{0}^{t} \int_{R_{v}} v\left[f_{h}^{2}(1, v, s)-f_{h}^{2}(0, v, s)\right] d s d v \\
& =\left\|f_{I}-f_{p}\right\|_{X}^{2} .
\end{aligned}
$$

The boundedness follows from the fact that the right hand side of this equation is independent of $t$, and that the second term on the left side is nonnegative. This immediately implies the asymptotic result. ing.

The above propositions have verified the follow-
Theorem 3.5 Assume $f_{I}-f_{0} \in D(\Omega)$ where $f_{I} \in X$ with $f_{0} \in X$ a given solution of Eq. (3.3) such that $v\left(\partial f_{0} / \partial x\right) \in X$ and $f_{0}$ satisfies the boundary conditions (2.4) and (2.5). Then Eq. (3.1) has a unique, global strong solution of the form (3.11) with $f_{p}=f_{0}$. Further, $f(\cdot,, t) \rightarrow f_{0}$ in $X$ as $t \rightarrow \infty$.

As pointed out earlier, these results all hold also in the (Brillouin) Case 2, if $X$ is replaced by $X_{B}=L^{2}([0,1] \times B)$ in which case the current and density also exist.

\section{THE STATIONARY WIGNER EQUATION}

Theorem 3.5 assumes the existence of a solution $f_{0}$ of the stationary Wigner equation with inflow boundary conditions such $f_{0} \in X$ and $v\left(\partial f_{0} / \partial x\right)$ $\in X$. We recall that for simplicity we are considering potentials which are polynomials of degree $N$ on $[0,1]$ with arbitrary extension to the exterior. Without loss of generality we may assume $V(0)=0$.

The equation we consider is (3.3) subject to inflow boundary data, i.e.,

$$
\begin{array}{ll}
f_{0}(0, v)=f_{+}(v), & v>0 \\
f_{0}(1, v)=f_{-}(v), & v>0
\end{array}
$$

with $f_{ \pm} \in L^{2}\left(\mathbb{R}_{v}\right)$. As already noted, there are infinitely many formal solutions of this problem, all of which may be constructed as the Wigner transform [4] of a function of the hamilitonian

$$
H=-\frac{1}{2} \frac{d^{2}}{d x^{2}}+V(x) .
$$

(See Ref. [17]). This means that

$$
f_{0}=[g(H)]_{w}(x, v)
$$

for some real function $g$; the subscript $w$ means "Wigner transform." Note that $[g(H)]_{W} \neq g\left(H_{w}\right)$ in general.

If $g(H)$ is expanded in a formal power series, then a formal solution would be

$$
f_{0}(x, v)=\sum_{n \in \mathbb{N}_{0}} \alpha_{n}\left[H^{n}\right]_{w}(x, v)
$$


with real coefficients $\alpha_{n}$. To obtain a solution to our problem, it is necessary to find coefficients $\alpha_{n}$ such that the series converges (in $X$ ) and the inflow boundary conditions are fulfilled.

We begin by computing $\left[H^{n}\right]_{w}$. This is done as follows. We first recall the formula [21] for the Wigner transform of the product of two operators $A B$ in terms of $A_{w}$ and $B_{w}$ :

$$
[A B]_{w}=A_{w} e^{\frac{\Lambda}{\mathrm{e}^{2}}} B_{w}
$$

where $\Lambda$ is the Poisson bracket operator

$$
\Lambda=\overleftarrow{\partial_{v}} \overrightarrow{\partial_{x}}-\overleftarrow{\partial_{x}} \overrightarrow{\partial_{v}}
$$

The arrows indicate the direction in which the derivatives operate. For example

$$
A_{w} \Lambda B_{w}=\left\{A_{w}, B_{w}\right\}_{P B}=\partial_{v} A_{w} \partial_{x} B_{w}-\partial_{x} A_{w} \partial_{v} B_{w} .
$$

Then

$$
\begin{gathered}
{\left[H^{n}\right]_{w}=H_{w} \mathrm{e}^{\frac{\Lambda}{2 i}}\left[H^{n-1}\right]_{w}=\left[H^{n-1}\right]_{w} \mathrm{e}^{\frac{\Lambda}{2 i}} H_{w}} \\
=H_{w} \mathrm{e}^{-\frac{\Lambda}{2 i}}\left[H^{n-1}\right]_{w} ;
\end{gathered}
$$

note [4] [21]

$$
H_{w}=\frac{1}{2} v^{2}+V(x) .
$$

This leads to the crucial formula

$$
\left[H^{n}\right]_{w}=H_{w} \cos \frac{\Lambda}{2}\left[H_{w}^{n-1}\right]=\left[H_{w}\right]^{n-1} \cos \frac{\Lambda}{2} H_{w} .
$$

Because of the form of $H,\left[H^{n}\right]_{w}$ is a finite sum, so the cosine and the exponentials in the above formulas are well defined.

Lemma 4.1 Equation (4.8) is equivalent to

$$
\left[H^{n}\right]_{w}=\left[H^{n-1}\right]_{w} H_{w}-\frac{1}{8} \frac{\partial^{2}}{\partial x^{2}}\left[H^{n-1}\right]_{w}+R_{M_{1}}
$$

with

$$
R_{M_{1}}=\sum_{k=1}^{M_{1}} \frac{(-1)^{k}}{(2 k) ! 2^{2 k}} \frac{\partial^{2 k} V}{\partial x^{2 k}} \frac{\partial^{2 k}}{\partial v^{2 k}}\left[H^{n-1}\right]_{w}
$$

where

$$
M_{1}=\min \left(n-1,\left[\frac{N}{2}\right]\right) .
$$

Remark Recall the potential is a polynomial of degree $N$.

Proof The proof is a simple process of differentiating, using the power series expansion of cosine and noting that in powers of $\Lambda$ cross terms do not contribute by virtue of Eq. (4.9).

Corollary 4.2 We have

$$
\left[H^{n}\right]_{w}=\sum_{j=0}^{n} C_{n j}(x) v^{2 j}
$$

where the $C_{n j}$ are $C^{\infty}$ on $[0,1]$.

Remark The $C^{\infty}$ property follows from the fact that $V$ is a polynomial. The same result would follow for any $C^{\infty}$ potential.

\section{Lemma 4.3 (Recursion formula).}

$$
\begin{aligned}
& C_{n l}=\left(V-\frac{1}{8} \frac{\partial^{2}}{\partial x^{2}}\right) C_{n-1, l}+\frac{1}{2} C_{n-1, l-1}+ \\
&+\sum_{k=1}^{M_{l+1}} \frac{(-1)^{k}}{(2 k) ! 2^{2 k}}(2 l+2 k)(2 l+2 k-1) \cdots \\
& \cdots(2 l+1) V^{(2 k)} C_{n-1, k+l} \\
& 0 \leq l \leq n
\end{aligned}
$$

with

$$
M_{l}=\min \left(n-l,\left[\frac{N}{2}\right]\right) .
$$

In particular

$$
\begin{aligned}
C_{n n} & =2^{-n} \\
C_{n, n-1} & =\frac{V}{2^{n-1}}+\frac{1}{2} C_{n-1, n-2}, \quad n \geq 2 \\
C_{10} & =V \\
C_{n l} & =0, \quad l>n .
\end{aligned}
$$


Proof The proof of (4.12) follows by induction using Lemma 4.1 and Corollary 4.2, collecting coefficients of powers of $v^{2}$. Note $C_{00}=1$ because $\left[H^{0}\right]_{w}=1$.

Corollary 4.4 The matrix $C$ with elements $C_{n l}$ is invertible; denoting $\gamma_{n l}=\left(C^{-1}\right)_{n l}$ we have $\gamma_{n n}=2^{n}$ and $\gamma_{n j}=0, j>n$.

Lemma 4.5 There is a constant $K$, depending only on $V$ and its (finite number of ) derivatives such that for any $n$

$$
\left|C_{n l}\right| \leq K^{n}, \quad l=0, \ldots, n
$$

Proof We use induction on $n$, starting with $C_{00}=1$. Let $K_{1}$ be the supremum of $C_{n-1, l}$ over $[0,1]$ and over all indices $n, l$ and $k$ appearing on the right hand side of (4.12) as coefficients (including the coefficients which appear in the second derivative term). There are only a finite number of such coefficients because $V$ is a polynomial and the maximum number of terms appearing in the sum is in (4.12) is uniformly bounded in $n$. Then $K=M K_{1}$ where $M$ is the maximum number of coefficients appearing in this process, which is also uniformly bounded in $n$.

Now assume that

$$
\left|\frac{\partial^{2 j} C_{n-1, l}}{\partial x^{2 j}}\right| \leq K^{n-1}, \quad 0 \leq 2 j \leq N .
$$

Then it is clear by the definition in (4.12) that

$$
\left|C_{n l}\right| \leq K^{n}, \quad 0 \leq 2 j \leq N .
$$

We now choose $K_{0}>\min \left(K, 2^{6}\right)$. Then it is clear by the definition in (4.12) that

Lemma 4.6 The elements $\gamma_{n j}$ of the matrix $C^{-1}$ satisfy the bound

$$
\left|\gamma_{n j}\right| \leq \frac{K_{0}^{n^{2}+\frac{1}{2}}}{\left(\frac{1}{2} K_{0}^{6}\right)^{n}}, \quad j=0,1, \ldots, n .
$$

Proof We observe that since $C$ is triangular, $C^{-1}$ can be computed by inverting the main minor matrices successively. Let $A_{n}$ be the inverse of the $(n+1) s t$ main minor matrix whose determinant is $2^{-(n+1)(n+2) / 2}$. Each $\gamma_{n j}$ contains $(n+1)$ ! products of the type

$$
C_{0 i_{0}} C_{1 i_{1}} C_{2 i_{2}} \cdots C_{n i_{n}}, \quad i_{j} \leq j
$$

where the sequence $\left(i_{0}, i_{1}, \ldots, i_{n}\right)$ is a permutation of the integers 0 to $n$. Using Lemma 4.5 this implies

$$
\left|\gamma_{n j}\right| \leq(n+1) ! 2^{\frac{(n+1)(n+2)}{2}} K^{n^{\frac{(n+1)}{2}}} .
$$

Using the elementary estimate

$$
K_{1}^{j} \geq j \log K_{1} \quad\left(\forall K_{1}>1\right) .
$$

We get from (4.13)

$$
\left|\gamma_{n j}\right| \leq \frac{\left(2 K_{1}\right)^{\frac{(n+1)(n+2)}{2}} K^{\frac{n(n+1)}{2}}}{\left(\log K_{1}\right)^{n}} .
$$

The result is implied by (4.16) with the choice $K_{1}=\frac{1}{2} K_{0}^{\frac{1}{6}}$.

Proposition 4.7 Choose $a>0$ and let $f_{+} \in L^{2}\left(\mathbb{R}_{v}\right)$ be the restriction to $v>0$ of a real analytic function of $v^{2}$ with expansion

$$
f_{+}(v)=\sum_{n=0}^{\infty} p_{n} v^{2 n}
$$

where the $p_{n}$ are such that

$$
\sum_{n=0}^{\infty}\left|p_{n}\right| K_{p}^{2 n^{2}}<\infty
$$

with $k_{p}=k_{0}$ (Lemma 4.6) if $a>4$ and $K_{p}=\max$ $\left(K_{0}, e^{\sqrt{2 / a}}\right)$ if $a \leq 4$. Then there exists a sequence $\left(\alpha_{n}\right)_{n \in \mathbb{N}_{0}}$ of real numbers such that

$$
f_{0}(x, v)=\sum_{n=0}^{\infty} \alpha_{n} \sum_{j=0}^{n} C_{n j}(x) v^{2 j}
$$

is a weak solution of (3.3) for $v>0$ satisfying the inflow boundary condition (3.17) at $x=0$, with the $C_{n j}(x)$ given by Lemmata 4.2, 4.3. Furthermore 
$f_{0} \in C^{\infty}\left([0,1] \times \mathbb{R}_{v}^{+}\right) \cap L^{p}\left([0,1] \times \mathbb{R}_{v}^{+} ; e^{-a v^{2}}\right) \quad$ and $f_{0} \in L_{l o c}^{p}\left([0,1] \times \mathbb{R}_{v}\right), \quad 1 \leq p \leq \infty$.

Proof Noting Eqs. (4.4) and (4.5), we see that (4.18) represents a formal solution of (3.3). To prove the proposition it is necessary to choose the $\alpha_{n}$ such that (4.18) converges to a function in $L^{p}\left([0,1] \times \mathbb{R}_{v} ; \mathrm{e}^{-a v^{2}}\right)$ and satisfies the inflow condition at $x=0$. This condition requires

$$
f_{+}(v)=\sum_{n=0}^{\infty} \alpha_{n} \sum_{j=0}^{n} C_{n j}(0) v^{2 j}, \quad v>0 .
$$

Introduce the vector notation

$$
\begin{aligned}
& \tilde{\alpha}=\left(\alpha_{n}\right)_{n \in \mathbb{N}_{0}} \\
& \tilde{v}=\left(v^{2 n}\right)_{n \in \mathbb{N}_{0}} \\
& \tilde{p}=\left(p_{n}\right)_{n \in \mathbb{N}_{0}} .
\end{aligned}
$$

Then

$$
f_{+}(v)=\tilde{p}^{T} \tilde{v}
$$

and the boundary condition is expressed as

$$
\tilde{p}^{T} \tilde{v}=\tilde{\alpha}^{T} C(0) \tilde{v}
$$

which implies

$$
\tilde{\alpha}^{T}=\tilde{p}^{T} C(0)^{-1} .
$$

Substituting into (4.18) gives

$$
f_{0}(x, v)=\tilde{p}^{T} C(0)^{-1} C(x) \tilde{v} .
$$

To prove the result, it is sufficient to show that the function $f_{1}(x, 0)$ defined by

$$
f_{1}(x, v):=\tilde{p}^{T} C(0)^{-1} C(x) \tilde{v}^{\frac{-a}{2} v^{2}}
$$

is bounded, since the other factor $\mathrm{e}^{-\frac{a}{2} v^{2}}$ gives the asserted $L^{p}$ properties. We use Stirling's formula in the form

$$
\frac{n^{n+\frac{1}{2}} \mathrm{e}^{-n}}{n !}=\frac{1}{2 \pi}\left(1+w_{n}\right), \quad \lim _{n \rightarrow \infty} w_{n}=0 .
$$

We consider the case $a>4$ ( $a<4$ is similar). Denoting the elements of the matrix $C(0)^{-1} C(x)$ by

$$
M_{n j}=\sum_{l=0}^{n} \gamma_{n l}(0) C_{l j}, \quad j \leq n
$$

We get, by Lemmata 4.5 and 4.6 , taking the appropriate supremum over $x \in[0,1]$

$$
\left|M_{n j}\right| \leq \frac{(n+1) K_{0}^{n^{2}+n+\frac{1}{2}}}{\left(\log \left(\frac{1}{2} K_{0}^{6}\right)\right)^{n}} .
$$

Substituting this estimate into (4.20) and using (4.21) twice, we find

$$
f_{1}(x, v) \leq D \sum_{n=0}^{\infty} \frac{\left|p_{n}\right|}{\sqrt{n}} \frac{K_{0}^{2 n^{2}}}{\left(\log K_{0}\right)^{2 n}\left(\frac{a}{2}\right)^{n}}
$$

which is finite by hypothesis. Here $D$ is a constant depending only on $K_{0}$, i.e., on the supremum of all the (finite number of ) derivatives of $V(x)$, $x \in[0,1]$. The $C^{\infty}$ property is obvious. The $L_{\mathrm{loc}}^{P}$ property follows from the above calculations without the weight function by restricting $v$ to a compact subset of $\mathbb{R}_{0}$.

Theorem 4.8 With $f_{+}$as in Proposition 4.7 and with similar assumptions on $f_{-}$, there exists weak solution $f_{0}(x, v)$ of Eq.(3.3) (which is classical pointwise) satisfying the inflow boundary conditions

$$
\begin{aligned}
& f_{0}(0, v)=f_{+}(v), \quad v>0 \\
& f_{0}(1, v)=f_{-}(v), \quad v<0
\end{aligned}
$$

with

$$
f_{0} \in C^{\infty}\left([0,1] \times \mathbb{R}_{v} \backslash\{0\}\right) \cap L^{P}\left([0,1] \times \mathbb{R}_{v} ; e^{-a v^{2}}\right)
$$

for all $a>0$, and $1 \leq p \leq \infty$.

Proof The proof proceeds as in Proposition 4.7 for the boundary at $x=1$. Then $f_{0}$ is constructed by joining the two solutions.

We denote by $B^{ \pm}$the sets of $\{v \in B \mid v \neq 0\}$, where we recall $B$ is the first Brillouin zone. The following theorems apply to Case 2 only. 
THEOREM 4.9 Let $f_{ \pm} \in L^{2}\left(B^{ \pm}\right)$be the restriction to $v>0$ and $v<0$ of a real analytic function of $v^{2}$ with expansion

$$
f_{ \pm}(v)=\sum_{n=0}^{\infty} p_{n}^{ \pm} v^{2 n}
$$

where the $p_{n}^{ \pm}$are such that

$$
\sum_{n=0}^{\infty}\left|p_{n}^{ \pm}\right| K^{2 n^{2}}<\infty
$$

with $k_{0}$ from Lemma 4.6 . Then there exist sequences of real numbers $\left(\alpha_{n}^{ \pm}\right)_{n \in \mathbb{N}_{0}}$ such that

$$
f_{0}^{ \pm}(x, v)=\sum_{n=0}^{\infty} \alpha_{n}^{ \pm} \sum_{j=0}^{n} C_{n j}(x) v^{2 j}, v \in B^{ \pm}
$$

is a weak solution of (3.3) (which is classical pointwise) subject to the boundary conditions

$$
\begin{aligned}
& f^{+}(0, v)=f_{+}(v) \\
& f^{-}(1,0)=f_{-}(v)
\end{aligned}
$$

and $[0,1] \times B^{ \pm}, \quad$ and $\quad f_{0}^{ \pm} \in L^{p}\left([0,1] \times B^{ \pm}\right)$, $1 \leq p \leq \infty$. The solution defined by $f_{0}=f^{+}$, $v \in B^{+}, f_{0}=f^{-}, v \in B^{-}$is a classical solution of (3.3) with $v \in B$ subject to the inflow conditions (4.22) and (4.23) on $[0,1] \times B \backslash\{0\}$ and $f_{0} \in L^{p}$ ([0, 1] $\times B), 1 \leq p \leq \infty$. Furthermore $v f_{0}, v\left(\partial f_{0} / \partial_{x}\right)$ and $\Theta f_{0} \in L^{p}([0,1] \times B), 1 \leq p \leq \infty$.

The proof follows from the $L_{\text {loc }}^{p}$ results of Theorem 4.8.

THEOREM 4.10 Define the current by

$$
I=-\int_{B} v f_{0}(x, v) d v
$$

Then

1. I is constant

2. I is unique

3. $I=-\int_{B^{-}} v f_{0}^{+}(1, v)-\int_{B^{-}} v f_{-}(1, v) d v$.
Proof

1. This follows from integration of Eq. (3.3) and noting $\int \Theta f d v=0$ (in both cases 1 and 2). See Eq. (1.10) of Ref. [13].

2. The proof is analogous to that of Proposition 2.4 .

3. This follows from the definition of the current and (1).

\section{Remarks}

1. Result of (3) of the above theorem indicates that the current could be calculated from $f^{+}$(or $f^{-}$) alone if these could be constructed independently .

2. The results of Theorem 4.10 also holds for Case 1 if appropriate regularity holds. Note that the density

$$
n=\int_{B} f_{B}(x, v) d v
$$

always exists in Case 2, but the analogous quantify in Case 1, involving an integration over $\mathbb{R}_{v}$, may not [13].

3. In principle, if $v$ is restricted to a bounded set (Case 2) the $x$ variable should be discrete. The hybrid limit used here, with $x$ continuous, is used also in Ref. [13], and some justification for it is given in Ref. [9], pp. 56-57. Case 1 is also a regularization and for small $a$ computations for Cases 1 and 2 would agree closely, with Case 1 current

$$
I_{a}=\int_{\mathbb{R}_{v}} v f_{0}(x, v) \mathrm{e}^{-a v^{2}} d v
$$

obeying an analogue of Theorem 4.10. The limit $a \rightarrow 0$ (or $B \rightarrow \mathbb{R}_{v}$ ) have not been shown to exist.

4. It is well known that weight functions like $\exp \left(-v^{2} / 2\right)$ appear in transport theory quite often, e.g., in the theory of the semi-classsical Boltzmann equation with measure-valued scattering rates in the collision operator $Q(F)$; it may happen that $Q$ has eigenvalues (e.g., zero) 
of infinite multiplicity with eigenspaces generated by functions of type $\exp \left(-v^{2} / 2\right) P\left(v^{2} / 2\right)$ for any polynomial $P$ satisfying some periodicity property (see Ref. [22]).

\section{Acknowledgment}

The authors are pleased to acknowledge useful discussions with Eugen Merzbacher, K.M. Case, John Dorning and Mark Buchanan. This research was sponsored by the U. S. National Science Foundation under Grant DMS-9403458. The first author was supported by a travel grant from DFG (Deutsche Forschungsgemeinschaft).

\section{References}

[1] Frensley, W. R. (1987). Phys. Rev., B36, 1570.

[2] Shih, T.-T., Huang, H.-C. and Wu, G. Y. (1994). Phys. Rev., B50, 2399.

[3] Drexler, H. et al. (1994). Phys. Rev. Lett., 73, 2252; Medeiros-Ribeiro, G. et al. (1994). Appl. Phys. Lett., 66, 1767.

[4] Zweifel, P. F. (1993). Trans. Theory Stat. Phys., 22, 459.

[5] Brezzi, F. and Markowich, P. (1991). Math. Meth. Appl. Sci., 14, 35.

[6] Illner, R., Lange, H. and Zweifel, P. F. (1994). Math. Analysis Appl. Sci., 17, 349.

[7] Lange, H. and Zweifel, P. F. (1996). Nonlinear Analysis TMA, 26, 551 .

[8] Zweifel, P. F. and Bruce, Toomire (1997). Trans. Theory Stat. Phys., 26(4-5), 629-636.

[9] Markowich, P. A., Ringhofer, C. A. and Schmeiser, C. (1990). Semiconductor Equations, Springer, Wien, p. 65.

[10] Arnold, A. (1994). Math. Meth. Num. Anal., 7, 853-872; Ringhofer, C. Ferry, D. and Kluksdahl, N. (1989). Trans. Theory Stat. Phys., 18, 331.

[11] Kluksdahl, N., et al. (1989). Phys. Rev., B39, 7720.

[12] Markowich, P. A. and Ringhofer, C. A. (1989). ZAMM, 69, 121.

[13] Degond, P. and Markowich, P. A. (1990). Math. Modelling Num. Analysis, 24, 695.

[14] Ben Abdallah, N., Degond, P. and Markowich, P. A. (1997). ZAMP, 48, 135.

[15] Arnold, A. (1995). "Self-consistent Relaxation-Time Models in Quantum Mechanics" Tech. Report, \#263; "The Relaxation-Time Wigner Equation", Tech. Report, \#254 (1994); Center for Appl. Math., Dept. of Math., Purdue University.

[16] Bernstein, I. B., Greene, J. M. and Kruskal, M. D. (1957). Phys. Rev., 108, 546.

[17] Lange, H., Toomire, B. and Zweifel, P. F. (1996). Trans. Theor Stat. Phys., 25, 713.

[18] Buchanan, M. and Dorning, J. J. (1993). Physics Lett. A, 179, 306; Buchanan, M. and Dorning, J. J. (1993). Phys. Rev. Lett., 70, 3732.
[19] Ziman, J. M. (1962). Electron and Phonons, Oxford Press, London; Ashcroft, N. and Mermin, W. (1976). Solid State Physics, Saunders College, Philadelphia.

[20] Pazy, A. (1983). Semigroups of Linear Operators and Applications to Partial Differential Equations, Springer, Berlin.

[21] Imre, K., et al. (1967). J. Math. Phys., 8, 1097.

[22] Majorana, A. (1992). "Space homogeneous solutions of the Boltzmann equation describing electron-phonon interactions in semi-conductors". Preprint University of Catania.

\section{A. APPENDIX}

The Relaxation-time Approximation The relaxation-time model adopted in this paper does not conserve charge. This can be seen by integrating Eq. (3.1) over velocity (assuming appropriate regularity) to obtain

$$
\frac{\partial n}{\partial t}+\frac{\partial I}{\partial x}=\frac{n_{0}-n}{\tau}
$$

where $n$ is the density and $n_{0}$ is the density corresponding to $f_{0}$. Charge conservation would require the right-hand side of (A1) to be equal to zero. A model which does conserve charge is given in Ref. [9], p. 34:

$$
\begin{aligned}
& \frac{\partial f}{\partial t}+v \frac{\partial f}{\partial x}+i \Theta(V) f \\
& \quad=\frac{1}{\tau}\left(f_{0}(x, v) \int f(x, v, t) d v-f(x, v, t) \int f_{0}(x, v) d v\right) .
\end{aligned}
$$

(Actually, in Ref. [13] $f_{0}$ is taken independent of $x$ ). This model clearly does conserve charge, but the results obtained in the present paper for the simpler relaxation-time model have not been proved for this more general model. This may be the subject of a future paper.

The rationale for a non-charge-conserving model is the following intuitive model. At time $t$, an electron is "absorbed" from the ambient distribution, held in captivity for a time $\tau$, after which it is re-emitted. Since the ambient density is different at times $t$ and $t+\tau$, the number of electrons absorbed are not balanced by the 
number re-emitted, which were absorbed at an earlier time.

\section{Authors' Biographies}

Horst R. Lange is Professor of Mathematics at Univeristaet Koeln. His current research interests lie in the theory of nonlinear partial differential equations (especially in nonlinear Schroedinger equations), and in those nonlinear partial differential equations which appear in quantum theory (especially in quantum semiconductor theory).
Bruce Toomire has recently completed his $\mathrm{Ph}$. D. in mathematical physics at Virginia Tech, where he has taught in both the mathematics and physics departments. Currently he is serving as instructor of physics.

Paul Zweifel is retired from Virginia Tech where he was University Distinguished Professor and Director of the Center for Mathematical Physics and Transport Theory and Chairman of the Mathematical Physics program of graduate studies. As an emeritus professor, he continues his research activities in transport theory. 

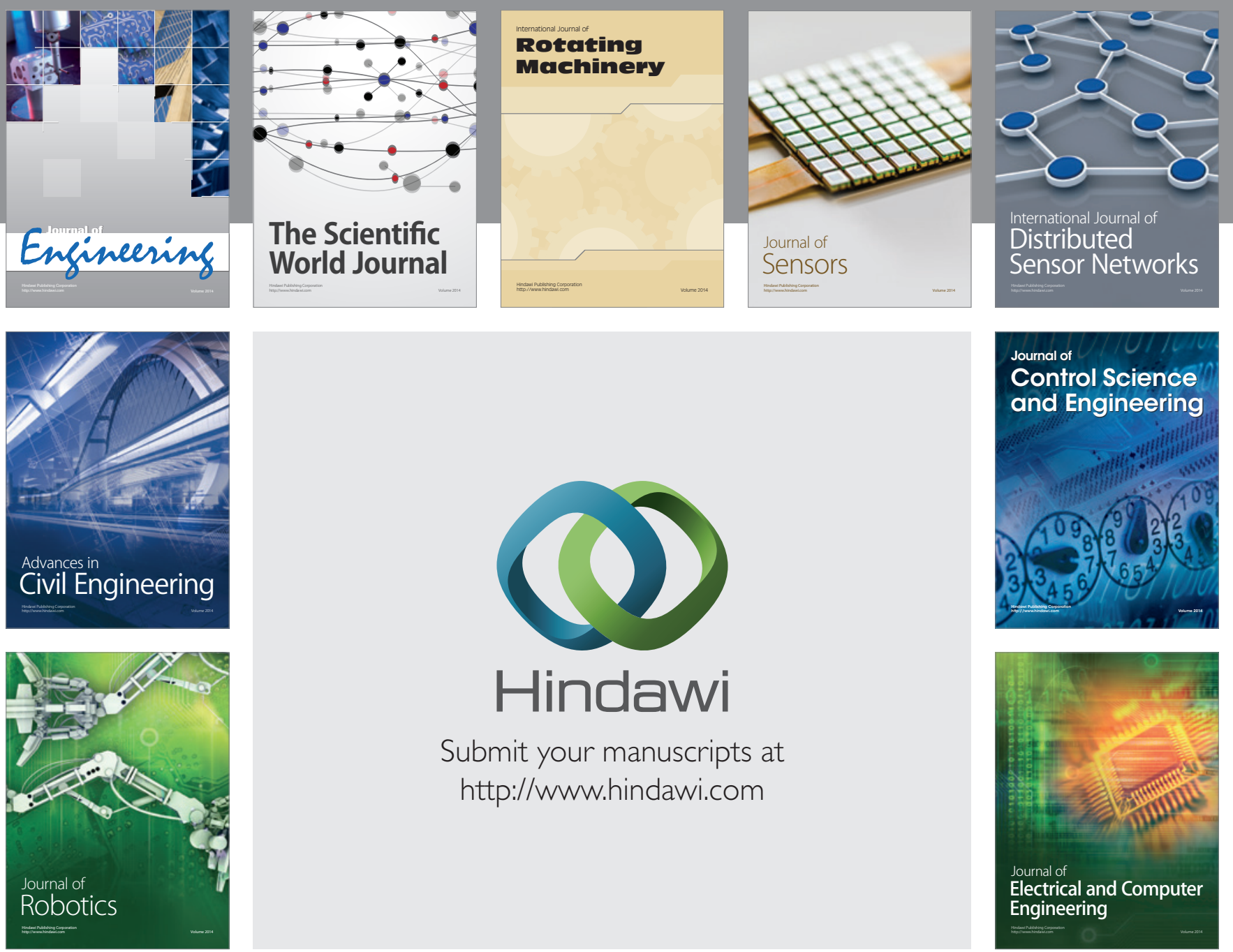

Submit your manuscripts at

http://www.hindawi.com
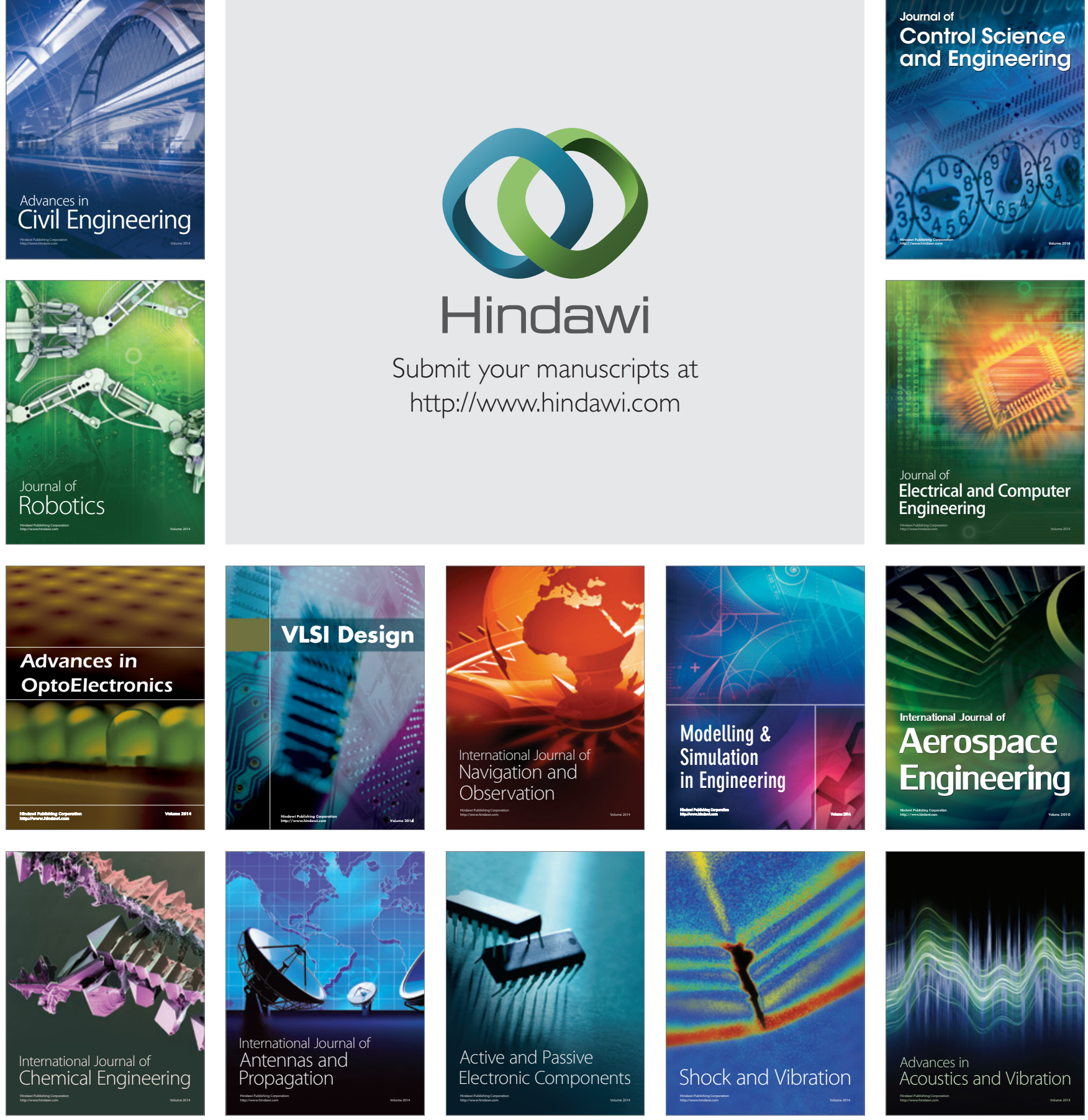